\title{
Variations of Moisture Content in The Presence of Combined Flux
}

\author{
Alpna Mishra ${ }^{1}$ and Sanjeev Kumar ${ }^{2}$ \\ ${ }^{1}$ Department of Mathematics, School of Basic Sciences and Research, Sharda University, Greater Noida-(India) \\ ${ }^{2}$ Department of Mathematics, Dr. B.R. Ambedkar University, Agra(India) \\ 1alpnamishra@gmail.com, ²sanjeevibs@yahoo.co.in
}

\section{ABSTRACT}

A model for diffusion in grains, through the drying process in the form of moisture removal, discussed through this work. While in drying, the moisture leaves the product as vapour or gas. This is achieved by impairing energy to the moisture molecule or changing the environment, so that the molecule will have sufficient latent energy to leave the product. Hence it is the process, which is intended to remove moisture from a feed substance so that the feed becomes "dry" as final product. Moisture reduction can also be achieved by mechanical methods. Using the pressure gradient as the driving force with combined flux, we get a system of partial differential equation which will be then solved with the help of MATLAB 7.0 and finally the graphs shows the variation of moisture with respect to diffusion coefficient at different temperature and as well as variation of moisture with the time.

Keywords: Equilibrium Moisture Content, Grain Moisture Content, Air Drying, Kiln Drying, Monolayer Values, Energy Constant.

AMS subject classification: $92 \mathrm{~B} 05$

Language: English

Date of Submission: 2018-03-24

Date of Acceptance: 2018-04-20

Date of Publication: 2018-04-30

ISSN:2347-1921

Volume: 14 Issue: 01

Journal: Journal of Advances in Mathematics

Website: https://cirworld.com

This work is licensed under a Creative Commons Attribution 4.0 International License. 


\section{INTRODUCTUION}

The removal of water or any solvent from a solid, semisolid or liquid through evaporation which generally remove its moisture content, is a mass transfer process and it is known as drying process. Seasoning of wood is a drying process as nearly as possible to the moisture content so that it will be attained in use or in other woods, to suitable moisture content warranting equilibrium with the prevailing atmospheric conditions in service. When wood is dried, surface zones dry in advance of the interior because of direct evaporation of the moisture at the surface. As a result, moisture gradient is a setup which causes the moisture, to move from the interior to the surface. The rate of drying of wood depends on various factors such as temperature, relative humidity, air circulation etc. Also, wood is dried by so many general methods that are available for wood drying.

In paddy grain, moisture is present at two places, first one at the surface of the grain which is known as surface moisture, while another in the kernel known as internal moisture. The surface moisture will readily evaporate when grain is exposed to hot air while internal moisture evaporates much slower, because first it has to move from the kernel to the outside surface, and as a result, surface moisture and internal moisture evaporate at a different rate. This difference, results in a different drying rate, which is then defined as the rate at which grain moisture contain declines during the drying process.

A drying curve shows how the grain moisture content $(\mathrm{MC})$ changes over time and how grain temperature changes. Also, drying rate is not constant but changes over time. Carlsson et al [1] shows that total drying time can be minimized within the acceptable limits at the same time, as both the moisture content and the deformation after completion of drying. The stress levels during drying were also considered and in this case modeling simulation and control of the wood drying process, in which an internal model was proposed in a non-linear system of a few partial differential equations by Tarasiewicz et al [2]. The initial conditions are calibrated by solving the static model and by the technical experiment. The moisture loss from wood products during drying was described by Dincer [3] and this study deals with the development of an analytical techniques for determining the moisture diffusivities and moisture transfer coefficients for wood products subjected to drying. Again, Tarasiewicz et al [4] presented the dynamic characteristics of the wood drying process by an actuator network and by an internal model and these two subsystems are composed of a set of ordinary differential, partial differential and algebraic equations with adjusting operating functions.

The two parameter BET (Brunauer-Emmett-Teller) and the three parameter GAB (Guggenheim-AndersondeBoer) isotherms (Where the (GAB) isotherm equation accurately describes measured soil water vapour sorption isotherms for a wide water activity range, and is suggested as a replacement for the commonly applied BET equation for SA estimation) was analysed by Timmermann [5] and they showed experimentally, that it is found that GAB monolayer value is always higher than the BET value while the GAB energy constant results always lower than the BET constant. The mathematical and physical reasons were also given, which explain these differences. A new model for thermodynamic analysis of a drying process in terms of energy was again given by Dincer et al [6] in which the energy efficiencies are derived as the functions of heat and mass transfer parameters. An illustrative example was also considered to verify the presented model in this work and to illustrate the applicability of this model to actual drying processes at different drying temperatures, specific energies of drying air, energy difference of inlet and outlet products, products weights, moisture contents of drying air, and humidity ratios of drying air.

The model was a mathematical one treating wood as a continuous and homogeneous medium and as far as the numerical approach is concerned, the finite element method was used. Decomposition of moist wood is modelled using the shrinking unreacted core approximated for a finite rate of reaction and the assumption of a thermally controlled evaporation of moisture across an infinitely thin front at constant temperature was given by Galgano et al [7]. A model for isothermal diffusion of bound water was used to simulate the thin layer drying kinetics of amaranth grain which was discussed by Resio et al [8]. The model assumed that the driving force for the transport of bound water is the gradient of spreading pressure. The gradient of spreading pressure was related to the moisture gradient using the GAB isotherm. The derivation of the GAB adsorption equation from 
the BDDT adsorption theory was showed by the Curie [9]. In general, the BET like adsorption equation without changes to the bulk liquid properties of multilayer molecules.

Effects of the drying temperature and moisture content on rice taste quality was shown by Zheng et al [10], in which the influence of drying temperature on rice taste quality was studied. Optimum safe drying temperature was identified by Oyoh et al [11] and it was shown that samples of selected fresh grains were dried in a grain dryer at different temperatures. The result from research indicates that the maximum drying temperature range for seed grains is between the ranges of 313-323 $\mathrm{k}$.

The effective moisture diffusivity values aromatic Pandanus leaves are determined in Rayaguru et al [13]. It also explains the effect of low RH was prominent during the initial drying when the product was moist, and the effect of temperature was prominent in the later part of drying, which acted as a driving force for moisture diffusion and hence the total drying time was reduced.

Feng et al [15] defined the effect of water on the quality of dried products is reviewed in light of recent applications of novel characterization methods (nuclear magnetic resonance (NMR), X-ray spectroscopy, and Raman spectroscopy) in quantifying the state and mobility of water in dried products. The drying kinetics models, especially those related to the quality loss, are also reviewed.

Jonathan et al in [16] describe a three-dimensional (3D) numerical study of the convective thermal transfer during forced-air drying of brown rice grains with the inclusion of moisture evaporation. Three levels of temperature were tested across a range from 40 to $60^{\circ} \mathrm{C}$. The objective of the study was to determine the temperature distributions in the drying. The drying kinetics models, especially those related to the quality loss, are also reviewed by Rafael et al [17].

In this paper we report a system of partial differential equation and to analyses the variations of moisture contents, which will be then solved and shown graphically.

\section{THE MATHEMATICAL DEVELOPMENT}

Drying is classically defined as a process that removes a liquid, usually water from a solid in equipment termed as dryer and there remains a wide application of various technologies to moisture removal. Although drying is differentiated from the dewatering, in which the moisture leaves the product as a vapour or gas. This is achieved by imparting energy to the moisture molecule. So that the drying process is intended to remove moisture from a feed substance and thereby condition the feed into "dry" final product, but it does not imply that the product will have no moisture content.

Suppose that the spreading pressure which is, usually denoted by $p_{0}$ defines the gradient for the diffusion of bound water, and it is related with the surface free energy by the following expression

$$
d p_{0}=C_{0} R T d\left(\ln a_{0}\right)
$$

where $C_{0}$ is the surface concentration of water, $R$ the gas constant, $T$ the absolute temperature and $a_{0}$ the water activity, defines the measure of the water in any substance available.

If, $\mathrm{m}_{\mathrm{o}}$ denotes the moisture content, $\mathrm{M}$ the molecular weight of water and $\mathrm{A}$ the specific surface area of the solid, then we have the following expression for the relationship between water surface concentration and moisture content,

$$
C_{0}=\frac{m_{0}}{M A}
$$


After substituting equation (2) into (1), we have,

$d p_{0}=\frac{m_{0}}{M A} R T d\left(\ln a_{0}\right)$

Integrating equation (3) within the limits 0 to $a_{0}$,

$p_{0}=\frac{R T}{M A} \int_{0}^{a_{0}} m_{0} d\left(\ln a_{0}\right)$

The rate of mass flow of water arises due to the spreading pressure will be given by the expression

$J=-r_{c} \rho m_{0} \frac{d p_{0}}{d r}$

so that the combined mass flux vector is given by

$N=J+\rho v$

where $r_{c}$ is the resistance coefficient, $\rho$ the solid density, $m$ the moisture content and $\frac{d p_{0}}{d r}$ spreading pressure gradient and finally the $\quad v=\sum_{\alpha=1}^{n}$ (mass average velocity, repogensents the mass fraction of any species and $\alpha$ as well the ${ }_{\alpha}$ velocity of species $\alpha$ with respect to a fixed coordinate.

After differentiating equation (3) we get,

$\frac{d p_{0}}{d r}=\frac{R T}{M A} \cdot \frac{m_{0}}{a_{0}} \cdot \frac{d a_{0}}{d m_{0}} \cdot \frac{d m_{0}}{d r}$

Substituting the result of equation (5) and (7) into equation (6),

$N=-r_{c} \rho \frac{R T}{M A} \cdot \frac{m_{0}^{2}}{a_{0}} \cdot \frac{d a_{0}}{d m_{0}} \cdot \frac{d m_{0}}{d r}+\rho v$

The equation of continuity in spherical shape is given as,

$\frac{\partial}{\partial t}\left(m_{0} \rho\right)+\frac{1}{r^{2}} \frac{\partial}{\partial r}\left(r^{2} N\right)=0$

where $\rho$ is the concentration of water in a porous solid.

Putting the value of $N$ from equation (8) in equation (9), we have

$\frac{\partial m_{0}}{\partial t}=\frac{1}{r^{2}} \frac{\partial}{\partial r}\left[r^{2}\left(D\left(m_{0}\right) \cdot \frac{d m_{0}}{d r}+v\right)\right]$, 
where $\quad D\left(m_{0}\right)=\frac{r_{c} R T}{M a} \cdot \frac{m_{0}{ }^{2}}{a_{0}} \cdot \frac{d a_{0}}{d m_{0}}$

Which shows dependency of diffusion coefficient on moisture and by evaluating $a$ and $d a_{0} / d$ rrom equation (11), the diffusion coefficient can be obtained from the GAB isotherm equation and as well from Pickett equation, then,

$$
\frac{m_{0}}{m}=\frac{C a_{0}\left(1-a_{0}\right)}{\left(1-a_{0}\right)\left(1-a_{0}+C a_{0}\right)}
$$

where $m$ is the monolayer moisture content and $C$ is the parameter,

Now by operating with equation (12) and substituting the results into equation (11) the following expression results, we get;

$D\left(m_{0}\right)=\frac{r_{c} R T}{M A}\left(\frac{C m K a_{0}}{1+(C-1) K^{2} a_{0}}\right)$

To solve equation (10) and (13) the following conditions should be assumed :

$$
\begin{aligned}
& m_{0}=m_{i} \quad \text { at } \quad t=0 \text { and } 0 \leq r \leq r_{0} \\
& m_{0}=m_{e} \quad \text { at } \quad t>0 \text { and } r=r_{0} \\
& \frac{\partial m_{0}}{\partial r}=0 \quad \text { at } \quad t>0 \text { and } r=0 \\
& v=0 \quad \text { at } \quad t=0 \text { and } \quad r=0
\end{aligned}
$$

\section{NUMERICAL RESULTS AND DISCUSSION}

Typically in drying there will be no change in the composition of the feed in physically and chemical manner, other than removing the moisture. The set of partial differential equations (10) and (13) along with initial and boundary conditions (14), are solved numerically through MATLAB 7.0, and the graphs shown through figure.1 to 4 , represents the variations of moisture content with respect to time and the radial distance for different values of monolayer moisture content and for the different $G A B$ parameters $(K, C)$. It can be observed very clearly that with the increase in time and increase in radius, the moisture get decreased. The two dimensional representation for the variation in moisture content with respect to radial distance is also shown in different Figures. The effect of combined flux on moisture can easily be shown through the graphs, which show minor variations in moisture due to the velocity and cannot effect the moisture variation at a large scale.

For solving the set of partial differential equation (10) and (13) with respect to equation (14), we are taking the following values of different parameters.

In the figure. 1 the radius has been taken as $1.29 \times 10^{-9}$ with the value of $G A B$ parameters as $C=11.34 \& \mathrm{~K}=0.82$. Also from figure. 2 onward, we have change the value of radius, and the GAB parameters $K, C$ and observed that there is proportionate declination in moisture with respect to time in all the cases.

$$
r_{c}=1.29 \times 10^{-9}, 1.36 \times 10^{-9}, 1.49 \times 10^{-9}, 1.78 \times 10^{-9}
$$



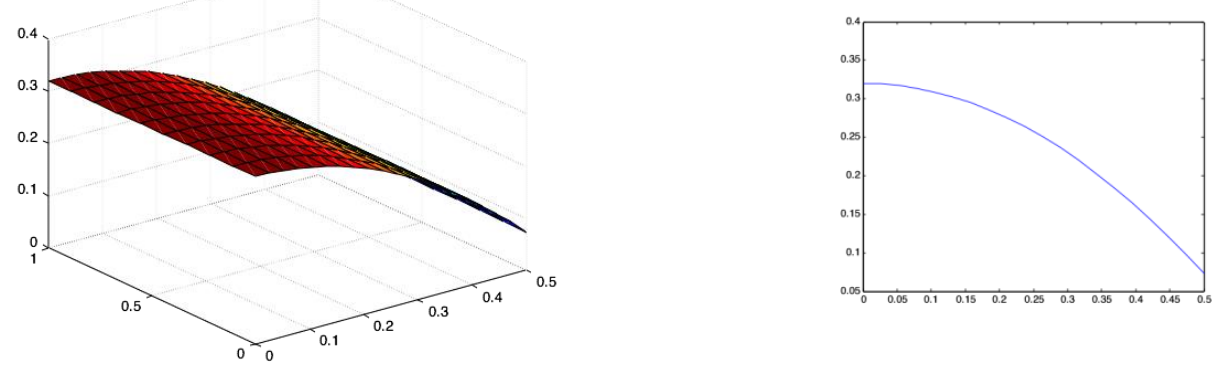

Figure.1: Variation of Moisture Content with respect to time (For K=0.82and C=11.34)
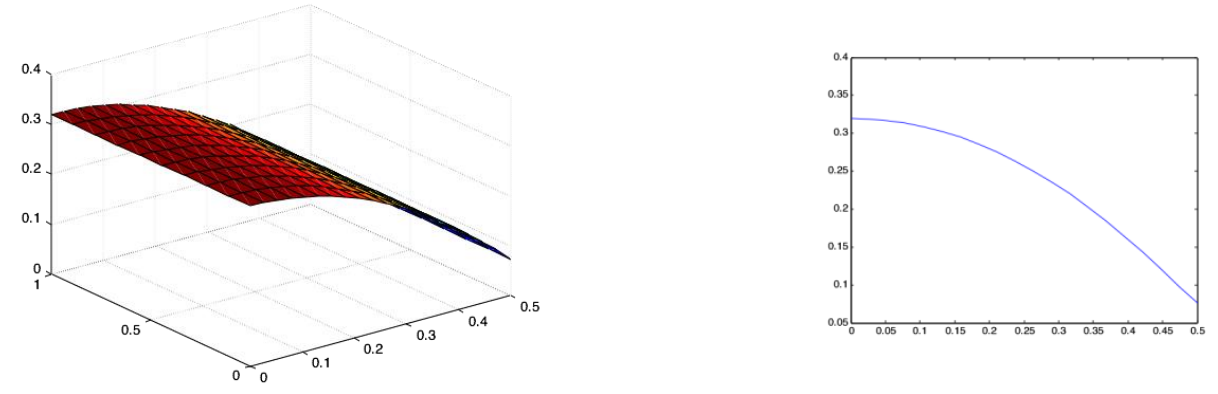

Figure.2: Variation of Moisture Content with respect to time (For $\mathrm{K}=0.80$ and $\mathrm{C}=9.89$ )
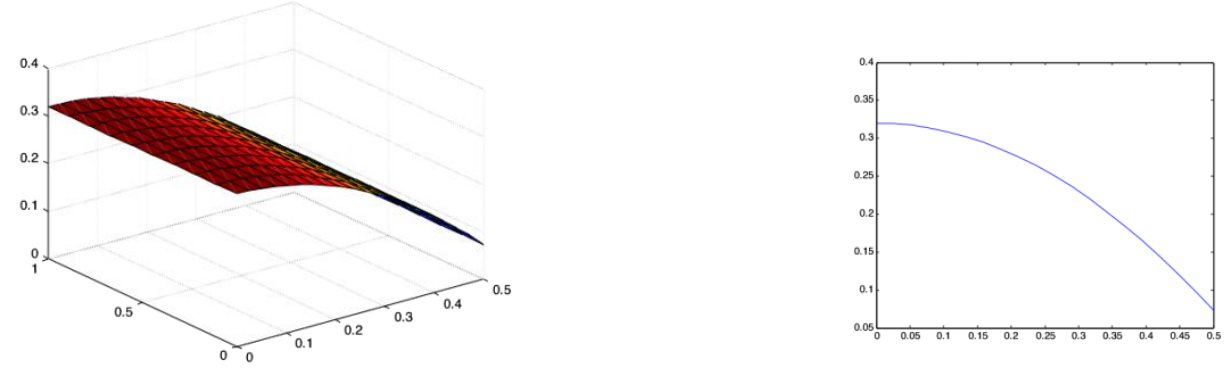

Figure.3: Variation of Moisture Content with respect to time (For K=0.78 and C=8.35)
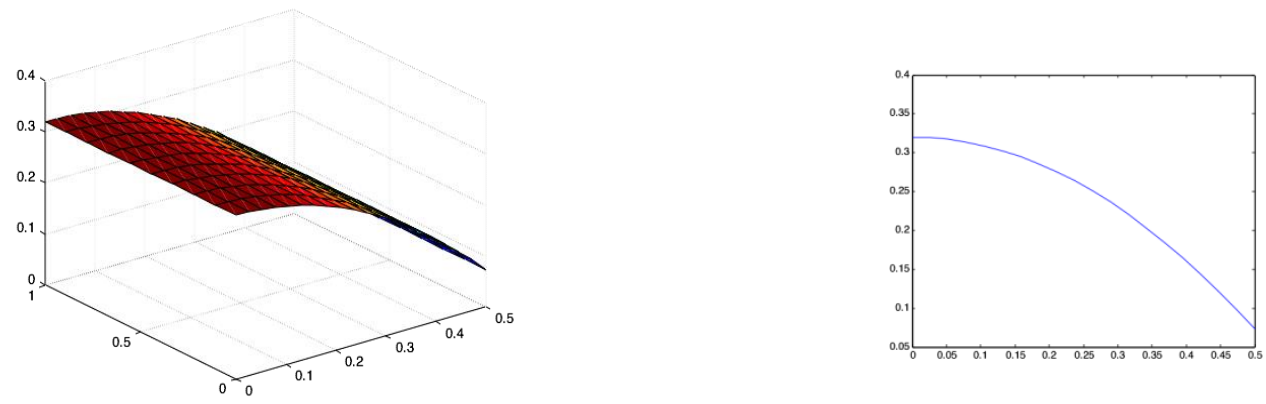

Figure.4: Variation of Moisture Content with respect to time (For K=0.75 and C=6.81) 


\section{REFERENCES}

1. Carlsson, P. and Esping, B. (1997): "Optimization of the wood drying Process", Structural Optimization, Vol. 141, pp-232-241.

2. Trasiewicz, S. and Leger, F. (1998): "Modeling simulation and control of wood", Drying Technology, Vol. 16(6), pp-1075-1084.

3. Dincer, I. (1998): "Moisture loss from wood products during drying", Energy Resources, Vol. 22(1), pp67-75.

4. Trasiewicz, S., Ding, F., Kudra, T. and Mrozek, Z. (2000): "Fast and slow generation of a multilevel control for the wood drying process", Drying Technology, Vol. 18(8), pp- 1709-1735.

5. Timmermann, E. O. (2003): "Multilayer sorption parameters: BET and GAB Values", Colloids and Surfaces A: Physicochem Eng. Aspect, Vol. 220, pp- 235-260.

6. Dincer, I. and Sahin, A. Z. (2003): "A new model for thermodynamic analysis of a drying process", International Journal of Heat and Mass Transfer, Vol. 47(4), pp- 645-652.

7. Galgano, A. and Colomba, D. (2004): "Modeling the propagation of drying and decomposition fronts in wood", Combustion and Flame, Vol. 139(2), pp-16-27.

8. Resio, A.C., Aguerre, R.J. and Suarez, C. (2005): "The drying of amaranth grain: Mathematical modeling and simulation", Brazilian Journal of Chemical Engineering, Vol. 22(2), pp-303-309.

9. Caurie, M. (2006): "The derivation of the GAB adsorption equation from the BDDT adsorption theory", International Journal of Food Science and Technology, Vol. 41, pp- 173-179.

10. Zheng, X. and Lan, Y. (2007): "Effects of drying temperature and moisture content on rice taste quality", Agricultural Engineering International: The CIGR E-journal, Vol. 9, pp-1-9.

11. Oyoh, K. B. and Menkiti, M. C. (2008): "Optimum safe drying temperature for seed grains", Agricultural Journal, Vol. 3(3), pp- 190-192.

12. Kumar, S. and Mishra, A. (2009): "Effect of transport ratio on source term in determination of surface emission coefficient", International Journal of Theoretical and Applied Sciences, Vol. 1(2), pp. 74-78.

13. Rayaguru, K. and Routray, W. (2010): "Effect of drying conditions on drying kinetics and quality of aromatic Pandanus amaryllifolius leaves", J Food Sci Technol, Vol. 47(6), pp-668-673.

14. Cai, L., Gao, D., Chen, T.B., Liu, H. T., Zheng, G. D. and Yang, Q. W. (2012): "Moisture variation associated with water input and evaporation during sewage sludge bio-drying", Bioresource Technology, Vol. 117, pp- 139.

15. Feng, L., Zhang, M. and Adhikari, A. (2014): "Effect of water on the quality of dehydrated products: A review of novel characterization methods and hybrid drying technologies", Drying Technology: An International Journal, Vol. 32(11), pp-1872-1884.

16. Jonathan, H. P., Fumina, T., Fumihiko, T., Daisuke, H. and Toshitaka, U. (2015): "Three-dimensional numerical modeling of convective heat transfer during shallow-depth forced-air drying of brown rice grains", Drying Technology: An International Journal, Vol. 33(11), pp-1350-1359.

17. Rafael, P. A., Mauri,F., Wanyr, R. F. and Gisele, T. S. (2017): "Energy and exergy efficiencies as design criteria for grain dryers", Drying Technology: An International Journal Vol. 36(4), pp-491-507. 